\title{
Spectral Classification and Luminosity Function of Galaxies in the Las Campanas Redshift Survey
}

\section{Citation}

Bromley, Benjamin C., William H. Press, Huan Lin, and Robert P. Kirshner. 1998. “Spectral Classification and Luminosity Function of Galaxies in the Las Campanas Redshift Survey." The Astrophysical Journal 505 (1): 25-36. https://doi.org/10.1086/306144.

\section{Permanent link}

http://nrs.harvard.edu/urn-3:HUL.InstRepos:41399833

\section{Terms of Use}

This article was downloaded from Harvard University's DASH repository, and is made available under the terms and conditions applicable to Other Posted Material, as set forth at http:// nrs.harvard.edu/urn-3:HUL.InstRepos:dash.current.terms-of-use\#LAA

\section{Share Your Story}

The Harvard community has made this article openly available.

Please share how this access benefits you. Submit a story.

Accessibility 
The Astrophysical Journal, 505:25-36, 1998 September 20

(C) 1998. The American Astronomical Society. All rights reserved. Printed in U.S.A.

\title{
SPECTRAL CLASSIFICATION AND LUMINOSITY FUNCTION OF GALAXIES IN THE LAS CAMPANAS REDSHIFT SURVEY
}

\author{
Benjamin C. Bromley and William H. Press \\ Physics Department, Harvard University; Harvard-Smithsonian Center for Astrophysics \\ HuAN LIN \\ Department of Astronomy, University of Toronto \\ AND \\ ROBERT P. KIRSHNER \\ Harvard-Smithsonian Center for Astrophysics \\ Received 1997 November 19; accepted 1998 April 3
}

\begin{abstract}
We construct a spectral classification scheme for the galaxies of the Las Campanas Redshift Survey (LCRS) based on a principal-component analysis of the measured galaxy spectra. We interpret the physical significance of our six spectral types and conclude that they are sensitive to morphological type and to the amount of active star formation. In this first analysis of the LCRS to include spectral classification, we estimate the general luminosity function, expressed as a weighted sum of the type-specific luminosity functions. In the $R$-band magnitude range of $-23<M \leq-16.5$ this function exhibits a broad shoulder centered on $M \approx-20$ and an increasing faint-end slope that formally converges on $\alpha \approx-1.8$ in the faint limit. The Schechter parameterization does not provide a good representation in this case, a fact that may partly explain the reported discrepancy between the luminosity functions of the LCRS and other redshift catalogs such as the Century Survey. The discrepancy may also arise from environmental effects such as the density-morphology relationship for which we see strong evidence in the LCRS galaxies. However, the Schechter parameterization is more effective for the luminosity functions of the individual spectral types. The data show a significant, progressive steepening of the faint-end slope, from $\alpha \approx+0.5$ for early-type objects to $\alpha \approx-1.8$ for the extreme late-type galaxies. The extreme late-type population has a sufficiently high space density that its contribution to the general luminosity function is expected to dominate at magnitudes fainter than $M=-16$. We conclude that an evaluation of type dependence is essential to any assessment of the general luminosity function.
\end{abstract}

Subject headings: galaxies: fundamental parameters - galaxies: luminosity function, mass function large-scale structure of universe - surveys

\section{INTRODUCTION}

Classification of galaxies has long been regarded as an important step toward understanding galaxy formation and evolution. There is considerable evidence that the two main methods of classification-morphological and spectralboth directly reflect physical properties of galaxies (Roberts \& Haynes 1994). Indeed it was clear early on that these two methods yield galaxy types that are strongly correlated (Morgan \& Mayall 1957), although recent work shows that the correlation is not perfect (Connolly et al. 1995; Zaritsky, Zabludoff, \& Willick 1995). In preparation for large galaxy catalogs such as the Sloan Digital Sky Survey efforts have begun to automate both approaches to classification. Morphologically based schemes must, of necessity, deal with pixel images, and the most promising technique employs artificial neural networks (e.g., Odewahn et al. 1996; Folkes, Lahav, \& Maddox 1996). Spectral schemes, on the other hand, require no more information than is already available in a typical redshift catalog. Furthermore, the interpretation of a spectral type is facilitated by the direct connection between spectral features and the physics of the stars and gas within the galaxies.

The information contained in a set of galaxy spectra can be extracted in an efficient manner by a principalcomponent method (e.g., Connolly et al. 1995). The idea is to identify features of the spectra that show the greatest variation from galaxy to galaxy, so that a classification scheme can reflect those physical properties that maximally distinguish galaxies. We here apply the principalcomponent method to analyze the galaxies in the Las Campanas Redshift Survey (LCRS), a large multiple-strip survey with over 25,000 measured spectra.

The goal of the work described here is not only to define spectral types but to begin discerning general physical properties of the galaxies in each type. We focus on the luminosity function, an important constraint for models of cosmic structure formation. We begin with a review of the LCRS catalog $(\S 2)$; in $\S 3$ we outline the principalcomponent method and compare our classification scheme with previous work. In $\S 4$ we present our analysis of the luminosity function according to spectral type.

\section{THE REDSHIFT DATA}

The Las Campanas Redshift Survey is described in detail by Shectman et al. (1996, hereafter LC1). Lin et al. (1996, hereafter LC2) provide additional details related to galaxy selection. We summarize characteristics of the survey briefly in this section.

The survey consists of $\sim 26,000$ galaxies in six sky strips, three strips in the northern Galactic cap region and three in the southern region. Each strip runs approximately $80^{\circ}$ across the sky in right ascension and has a width in decli- 
nation of $\sim 1.5$. The mean strip declinations are $-3^{\circ},-6^{\circ}$, and $-12^{\circ}$ in the northern sample and $-39^{\circ},-42^{\circ}$, and $-45^{\circ}$ in the southern sample.

Each sky strip was subdivided into 50 or so fields of square or nearly square dimensions. Galaxies in each field were selected on the basis of Kron-Cousins $R$-band magnitudes; a subset of these galaxies was chosen randomly for spectroscopic study using multiobject fiber spectrometers of either 50 or 112 fibers. Apparent magnitude limits vary from field to field, with typical isophotal limits of $16.0 \leq m<17.3$ and $15.0 \leq m<17.7$ for the 50 fiber and 112 fiber fields, respectively. Additional limits were imposed on the basis of the "central surface brightness" of the galaxies, corresponding approximately to the flux entering a fixed fiber aperture of 3.5 ; the limiting central magnitude is in the range of $m_{c}=18-19$, depending on the isophotal magnitude. This additional cut (a feature common to all magnitude-limited surveys, whether quantified or not) amounts to a $20 \%$ reduction in the fraction of galaxies in the 50 fiber fields and less than a $10 \%$ reduction of the 112 fiber fields. We emphasize that each field has assigned to it a unique set of parameters that include isophotal magnitude limits, central magnitude cut, and "sample fraction" of the total galaxies in that field for which spectra were observed. As in LC2, we take full account of these field-to-field differences.

The individual spectra range from 3350 to $6750 \AA$ for the 112 fiber data and from 3350 to $6400 \AA$ for the 50 fiber data. The wavelength resolution is $\sim 7 \AA$ (FWHM), and the sampling rate is $2.5 \AA$ per channel. As discussed below, we consider a narrower range of wavelengths to allow the redshifted spectra to be rescaled into a common waveband in the rest frame.

We work with the full catalog of 25,327 galaxies to perform the spectral classification in the next section. Following LC2, our analysis of the luminosity function in $\S 4$ makes use of only those galaxies with absolute magnitudes between -23 and -16.5 and with redshifts such that 1000 $\mathrm{km} \mathrm{s}^{-1} \leq c z<60,000 \mathrm{~km} \mathrm{~s}^{-1}$. We also exclude the 50 fiber data because of their more stringent surface brightness cuts. This leaves 18,278 objects, 8329 in the north and 9949 in the south. The same labeling scheme appears here as in LC2, so, for example, N112 refers to the northern 112 fiber data.

\section{SPECTRAL CLASSIFICATION}

Toward quantitative classification of the LCRS catalog we seek a representation of the spectra that accentuates the differences between individual galaxies. Such a representation is afforded by singular-value decomposition (SVD) as part of a principal-component analysis (see Kendall 1975). The overall strategy is to express the spectra in terms of a series of templates - following Connolly et al. (1995) we call them "eigenspectra" - and to select those that are most sensitive to galaxy-to-galaxy variations. The coefficient obtained by projecting a galaxy's spectrum onto such an eigenspectrum can serve as its spectral type. SVD gives the general prescription for how to build the eigenspectra; principal-component analysis demonstrates that they indeed characterize those properties of the galaxies that optimally distinguish them.

\subsection{Singular-Value Decomposition}

Let the vector $s_{i}$ represent the spectrum of the $i^{\text {th }}$ galaxy in a catalog of $N$ objects. The components of the vector, $s_{i j}$, are integrated fluxes in frequency channels, where $j$ is the channel index. The full catalog then is a matrix $S$ of size $N \times M$ where $N=25,327$ and the number of channels $M=800$. We first preprocess the spectra in this matrix by weighting each wavelength channel using a smoothed version of the mean spectrum in the observatory frame (this step downweights those wavelengths where the instrumental response is low). We then normalize the weighted spectra to some constant flux value. Next, the data are shifted into the rest frame and high-pass filtered to remove fluctuations in the spectra that have power on scales of $\sim 1000 \AA$ or more. Finally we subtract off the average of the filtered spectra-we want the power in the eigenspectra to reflect the differences between galaxies, not their similarities. It is this normalized, shifted, high-pass filtered entity that we call the catalog, $S$.

We can write the matrix $S$ in the form

$$
S=U W V^{T}
$$

where $U$ is a column-orthogonal $N \times M$ matrix and $W$ and $V$ are both $M \times M$; the former is diagonal with elements $w_{i} \geq 0$, and the latter is orthonormal (cf. Press et al. 1992, $\S 2.6$ ). This equation is the SVD of $S$. It is generally valid for all matrices provided that the number of columns does not exceed the number of rows, i.e., $N \geq M$.

Notice that any single spectrum $s_{i}$ from the catalog can be easily reconstructed from the matrices $U, W$, and $V$ in equation (1):

$$
\boldsymbol{s}_{i}=\sum_{j=1}^{M} \gamma_{i j} \boldsymbol{v}_{j}
$$

where $\gamma_{i j}$ is the $i j^{\text {th }}$ element of the product $U W$ and the vector $v_{j}$ is the $j^{\text {th }}$ column of $V$. This property highlights the special significance of $V$ : its columns define orthonormal vectors $v_{1} \ldots v_{M}$, the eigenspectra of which form the basis used in a principal-component analysis. The defining characteristic of such a basis is that it diagonalizes the covariance matrix of the catalog:

$$
\begin{aligned}
C & \equiv S^{T} S, \\
& =V W^{T} U^{T} U W V^{T}, \\
& =V W^{2} V^{T},
\end{aligned}
$$

(note that $U^{T} U=1$ ). Projecting the expressions in equation (3) onto the eigenspectra explicitly demonstrates that $C$ is diagonalized, leaving the eigenvalue equation

$$
C v_{i}=w_{i}^{2} v_{i}
$$

where $w_{i}$ is the $i^{\text {th }}$ diagonal element of $W$ in equation (1).

There may also be value in weighting individual data points differently, depending on measurement errors or prior assumptions about the data. Thus, before calculating the SVD, one may wish to write the elements of the catalog matrix as

$$
s_{i j} \rightarrow g_{i} h_{j} s_{i j},
$$

where $g_{i}$ and $h_{j}$ are weight factors. A transformation of this type can describe the effects of the "renormalization" that we performed on the spectra in the LCRS catalog.

A geometric interpretation of the eigenspectra is that they are aligned with the principle axes of the error ellipsoid corresponding to the scatter of points in the catalog. The eigenspectra with the largest eigenvalues (the $w_{i}^{2}$ in eq. [4]) indicate directions in the frequency-channel space where there is greatest variation between objects in the catalog. 
Conversely, small eigenvalues correspond to directions along which the data show little variation.

The idealization that data scatter is an error ellipsoid hints that a principal-component analysis is best suited to systems where the data correspond to $M$ statistically independent Gaussian variants. Knowledge of the covariance matrix allows the original orthogonal basis in which the data are collected (i.e., frequency channels) to be rotated into a new basis (the eigenspectra) to represent the data in terms of statistically independent coefficients (the $\gamma_{i j}$ in eq. [2]). This rotation is the Karhunen-Loève transform. In general, where higher order correlations are possible, these coefficients will not necessarily be statistically independent.

A data compression algorithm is evident from the geometrical interpretation above: Simply keep only the first $m$ eigenspectra $\boldsymbol{v}_{j}$ and coefficients $\gamma_{i j}$ that correspond to eigenvalues at or above a threshold $w_{m}^{2}$; all the rest correspond to modes along which the data show relatively small variation. Our galaxy classification method follows just this strategy.

The simplest scheme is to consider only $\gamma_{i 1}$, the dot product of the $i^{\text {th }}$ galaxy spectrum with the first eigenspectrum $v_{1}$. The galaxies have the greatest variance with this coefficient, and it alone may be sufficient to usefully define spectral type. Then the values of $\gamma_{i 1}$ could be partitioned into bins, with each bin corresponding to a unique type. Here, we use the first two eigenspectra (Fig. 1) to define spectral type because the geometry of data-point scatter in the vector space of frequency channels is not exactly elliptical; there exist correlations between coefficients such that the scatter of data points roughly forms an

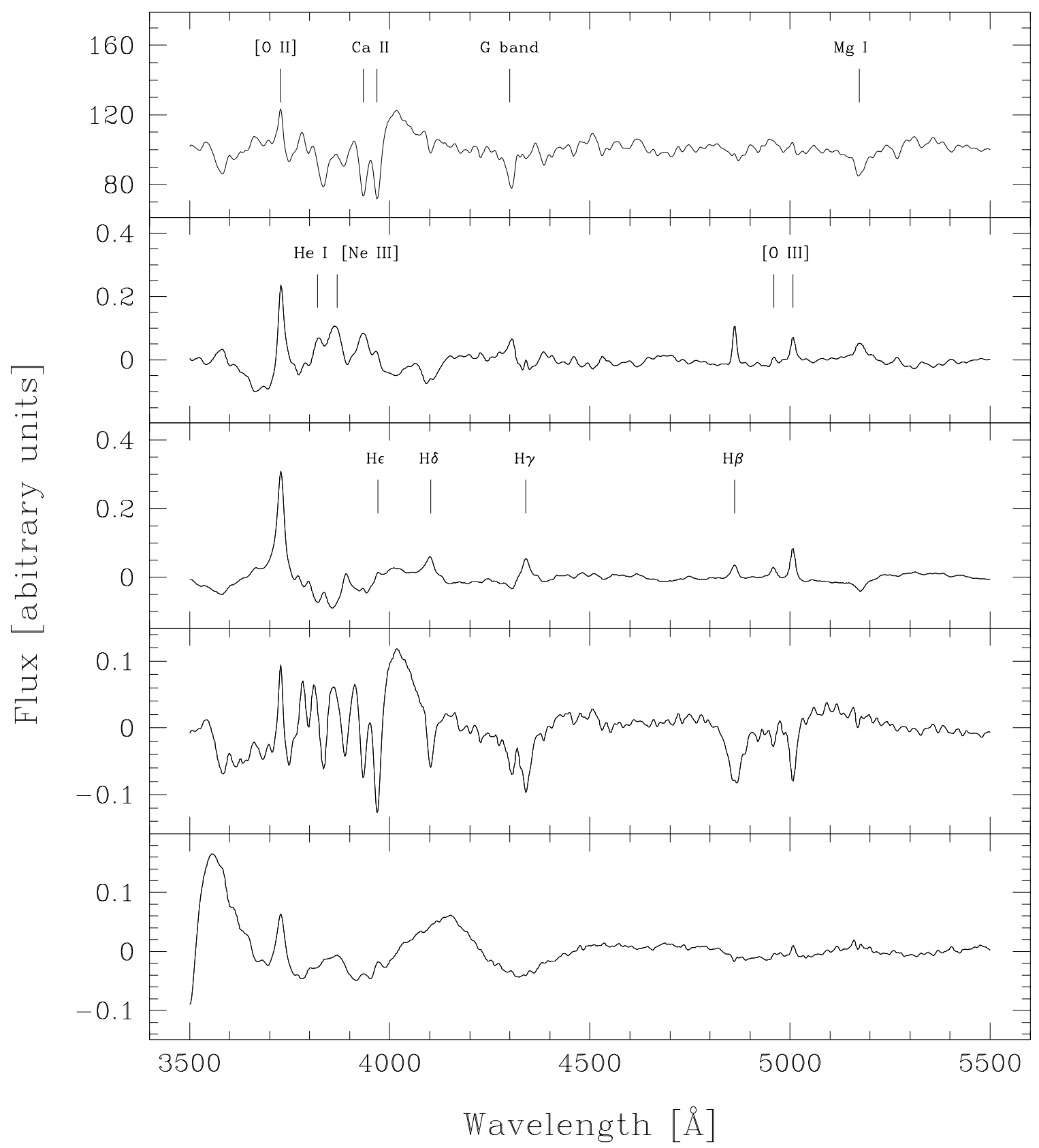

FIG. 1.-Mean spectrum of the LCRS galaxies after high-pass filtering (top) and the first four eigenspectra from SVD. The first eigenspectrum (which has the largest eigenvalue) is second from the top, the second eigenspectrum is immediately below it, and so forth. The wavelength of some atomic line features are marked; note that these features represent either emission or absorption, depending on the sign of the weight factor multiplying each eigenspectrum. 
arc. We create distinct types by extending boundaries radially outward from a point at some distance from the arc. Figure 2 illustrates the six spectral types-here called "clans"-defined in this way. The boundaries were chosen so that each clan contains roughly similar path lengths along a trajectory that runs along the central ridge of the arching scatter figure. In this sense the dimensionality of our classification scheme is one; however, because of the non-Gaussian nature of the data scatter, the classification parameter's trajectory lies in a two-dimensional projection of the principal-component eigenvectors.

The choice of clan boundaries as shown in Figure 2 was meant to capture the central peak in the density of the data scatter (clan 2) and the transition along the "tail" of the scatter diagram toward positive values of projection coefficients (clans $3-5)$. The remaining two clans (1 and 6) are defined to assess the outliers of the scatter diagram. We find evidence from the systematic trend in luminosity function shape with clan index $(\S 4.2)$ that the outlier clans represent populations of galaxies with unique properties, as opposed to random scatter of galaxies in neighboring clans.

Before analyzing astrophysical properties of the clan populations, we discuss qualitatively the line and continuum features that appear in the eigenspectra. This will help place our classification scheme in the context of morphological classification and other spectral classification methods.

\subsection{Physical Significance of the Eigenspectra}

Recall that in deriving the eigenspectra, we subtract the mean spectrum of the 25,327 galaxies. Thus, the eigenspectra measure differential signal in continuum and line features. Figure 1 contains the mean spectrum of the LCRS galaxies along with the first four eigenspectra. The first eigenspectrum shows emission features in $\mathrm{H}$ I, He I, O II, O III, $\mathrm{Ne}$ III, $\mathrm{Ca}(\mathrm{H}$ and $\mathrm{K})$, and $\mathrm{Mg} \mathrm{I}$, as well as slight continuum "absorption" on the red side of the $4000 \AA$ Balmer limit. Because the individual spectra are high-pass filtered, i.e., flattened, the $4000 \AA$ break shows up as a sawtooth-shaped

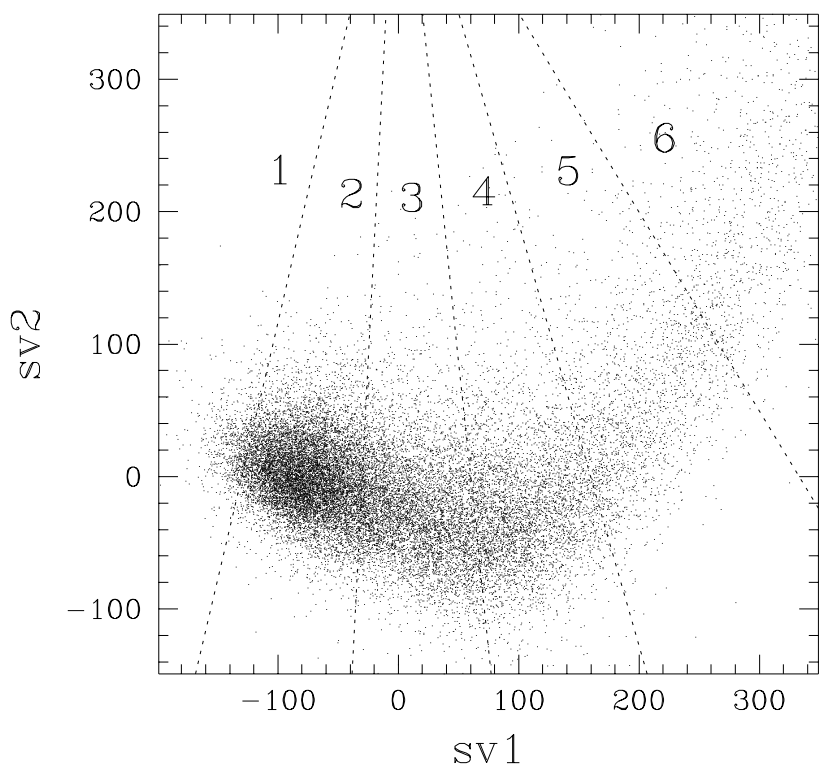

Fig. 2.-Scatter diagrams of the projection coefficients (sv1 and sv2) for the first and second eigenspectra in Fig. 1. The regions determined by the straight lines define the galaxy clans, labeled 1-6. profile. Some of the emission features $-\mathrm{H} \beta$ and forbidden $\mathrm{O}$ II and $\mathrm{O}$ III lines - are direct indicators of physical processes, presumably in the interstellar medium. Others, such as the $\mathrm{Ca} \mathrm{H}$ and $\mathrm{K}$ lines and $\mathrm{G}$-band complex lines, appear in "emission" primarily to compensate for spectra with weaker absorption than is exhibited in the mean spectrum.

Evidently, the most significant spectral variation between galaxies is the degree of line emission. Emission lines of oxygen and hydrogen Balmer lines are expected from $\mathrm{H}$ II regions, while the diminished stellar absorption in the calcium lines indicates a young stellar population. Therefore a positive projection coefficient from this eigenspectrum seems like a good indicator of star formation. Indeed, the most prominent feature in the eigenspectrum, the [O II] $\lambda 3727$ line, is known to correlate with star formation rate as well as morphological type (Kennicutt 1992a). However, there are two caveats. The first is that better measures of star formation are known, specifically $\mathrm{H} \alpha$ flux and the shape of the UV continuum, but both are outside of the waveband considered here. The second caveat is that it can be difficult to make a distinction between star-forming regions and active galactic nuclei (AGNs) or LINERs solely on the basis of the emission lines contained in the eigenspectrum. Again, $\mathrm{H} \alpha$ measurements would have helped (Baldwin, Phillips, \& Terlevich 1981; Allen et al. 1991). The fraction of objects with nonthermal spectra is expected to be small, and therefore we hold to the interpretation that emission lines associated with the first eigenspectrum indicate star formation. Below we present evidence (Fig. 4) that AGN spectra can be isolated, but we use the coefficient of projection with the third eigenspectrum.

The second eigenspectrum is primarily dominated by

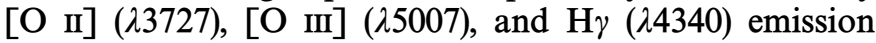
lines. It allows a fine-tuning of the line contributions in star-forming galaxies; there is a tendency for galaxy spectra that have a large projection coefficient with the first eigenspectrum to couple with the second eigenspectrum as well. This correlation is manifested by the curvature of the data scatter in Figure 2. The appearance of strong $\mathrm{H} \delta$ emission in the eigenspectrum suggests that it positively correlates with star-forming $\mathrm{H}$ II regions at relatively high levels of excitation.

Ultimately, the physical significance of the eigenspectra is best interpreted by examining the spectra that they represent. In Figure 3 we show the average spectra of objects in the six clans. A progression from clan to clan is clear and supports our interpretation that the most important spectral differences between galaxies lie in the amount of line emission.

While not relevant to our present spectral classification scheme, the third eigenspectrum is noteworthy. It has the role of further fine-tuning specific line-emission features, including [O II] $\lambda 3727$, as well as continuum emission above $4000 \AA$. Note that it shows strong absorption in $\mathrm{H} \gamma$ and $\mathrm{H} \delta$ and an asymmetry in the $\mathrm{Ca} \mathrm{H}$ and $\mathrm{K}$ troughs from $\mathrm{H} \epsilon$. The distribution of projection coefficients for this eigenspectrum is roughly symmetric except for a weak tail extending to large negative values, with a significant population-about $0.6 \%$ of the total-between 3 and 7 standard deviations. The mean spectrum of these objects is shown in Figure 4. The projection coefficients of the third eigenspectrum are negative for this population, hence it shows strong emission in the Balmer series. With the pronounced, broad Balmer lines and enhanced [O III $] /[\mathrm{O}$ II $]$, 


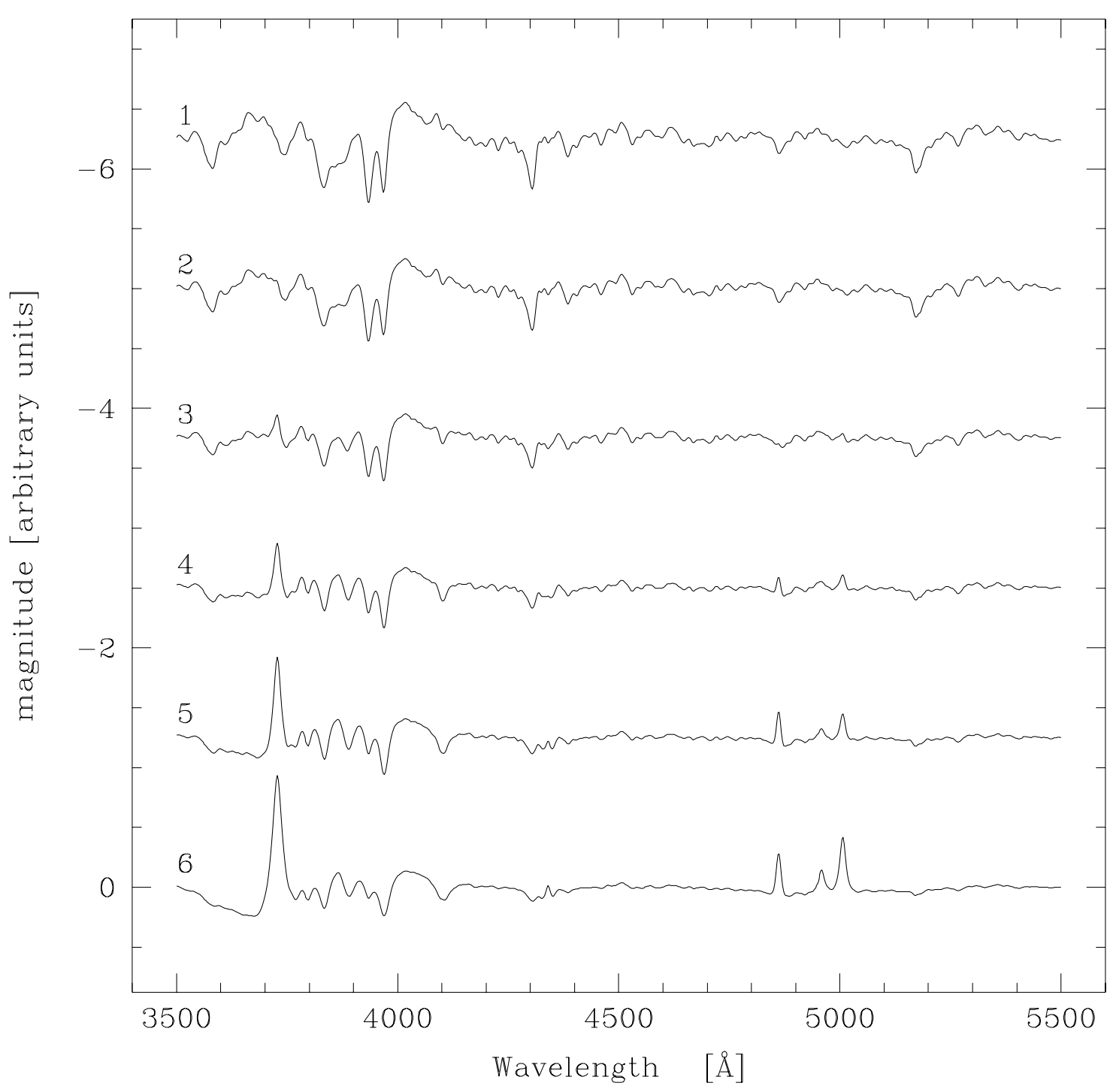

Fig. 3.-Mean spectra of the six clans. A low-frequency component has been removed, as in Fig. 1, and each curve has been offset for clarity. The labels indicate the clans as defined in Fig. 2.

we interpret these objects as Seyfert 1 galaxies (cf. Kennicutt 1992b).

The skewness in the distribution of projection coefficients steadily decreases with increasing eigenspectrum index. By the fourth eigenspectrum, the distribution is nearly symmetric (skewness is around 0.2 , as compared with 1.2 for the first eigenspectrum). This is reassuring because it suggests that the scatter in the higher order eigenspectra is becoming more and more characteristic of Gaussian scatter and contains less information relevant to classification.

\subsection{Comparison with Other Spectral Classification Schemes}

A variety of spectral classification schemes for galaxies has been proposed in the literature, and recent examples include Allen et al. (1991), Bershady (1995), Zaritsky et al. (1995), and Kurtz \& Mink (1997). Most of these methods involve fitting to specific line features or to a predefined spectral template that includes these features. Connolly et al. (1995) proposed a method that incorporates eigenspectra defined primarily from the data themselves in a strategy very similar to the one used here. We begin this section by considering their technique.

Connolly et al. performed a principal-component analysis of optical and UV data to classify galaxies according to the projection of their spectra onto eigenspectra. The eigenspectra were derived from a set of 10 templates, where each template is the average of spectra from a given morphological type or class of starburst galaxy. Since the number of frequency channels is large, over 2200 in their case, Connolly et al. properly construct their correlation matrix using the space defined by the 10 templates, not the space of frequency channels. Thus each "data point" is essentially a measure of how the flux is distributed among the 10 templates. Connolly et al. also assign a variable weight to each template (as in eq. [5]), but otherwise, the procedure is the same as in a "standard" principalcomponent analysis. Connolly et al. argue that their projection of the data onto the space of templates offers several advantages over the standard method, not the least of which is a reduction in computational load. In general, 


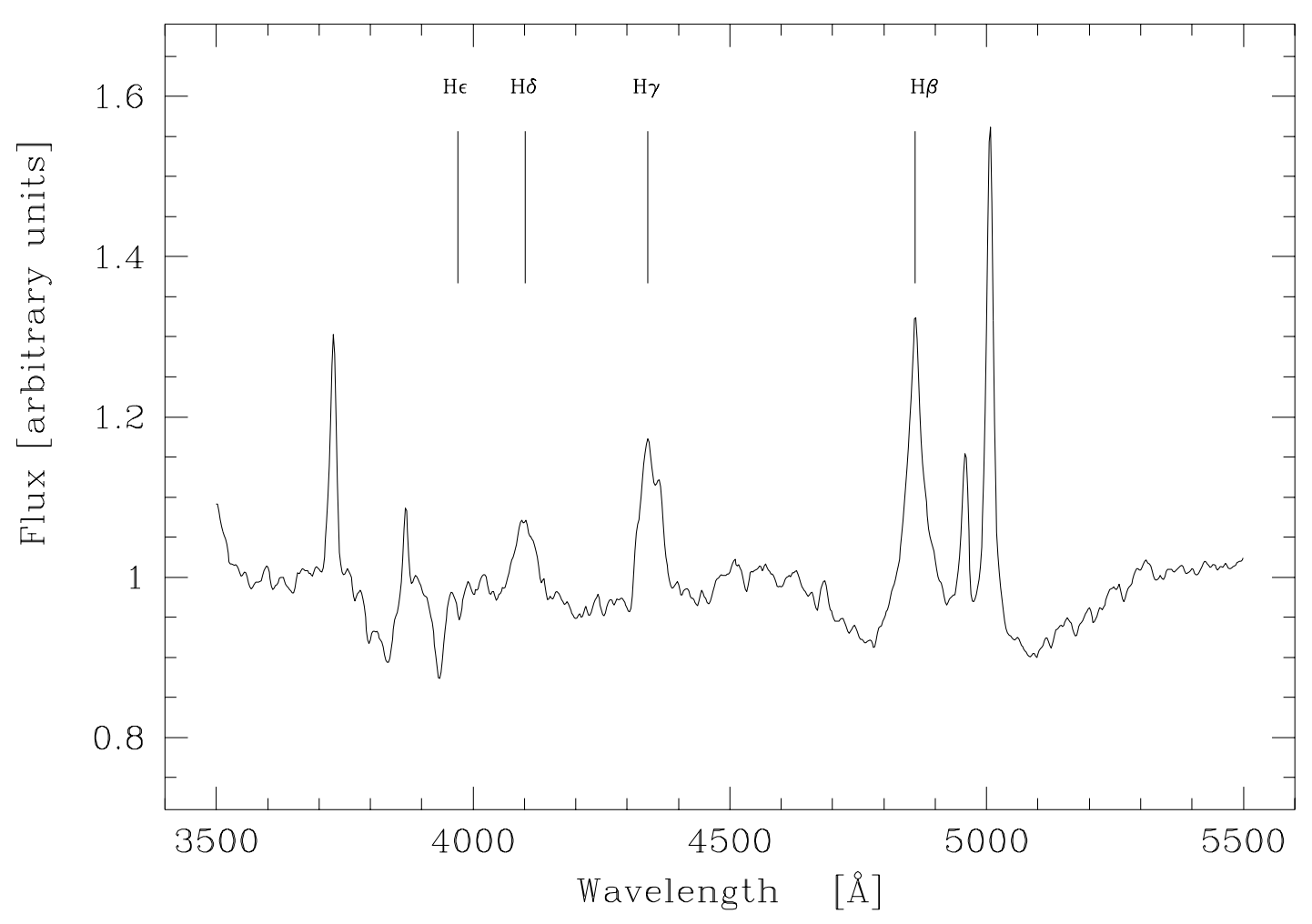

FIG. 4.-Mean spectrum of galaxies with abnormally strong, negative projection coefficients for the third eigenspectrum. As in Fig. 3, a low-frequency component has been removed. Strong Balmer emission is evident in the spectrum, and the interpretation here is that these are Seyfert 1 galaxies.

there is considerable savings in diagonalizing a $10 \times 10$ matrix versus one of size $2200 \times 2200$ (although of rank 10).

A nice way to view the differences between the method of Connolly et al. and a standard principal-component analysis is through the formalism of SVD. The raw data, in this case a $10 \times 2200$ matrix formed of 10 vectors in the 2200-dimensional space of frequency channels, are projected onto the 10-dimensional space of templates simply with a matrix transpose operator. Indeed, it is formally necessary to take the transpose of equation (1) to interpret the matrices $V$ and $W$ in terms of eigenspectra and eigenvalues as we did earlier - this enables the data of Connolly et al. to meet the requirement that the number of rows be greater than the number of columns. Numerically, it is not at all necessary to work with the transpose. One simply performs the SVD and pulls the eigenspectra out from the rows of the matrix $U$ in equation (1) (see Press et al. 1992, p. 66).

In practice, it may not be feasible to decompose a catalog matrix if the number of data points and the number of frequency channels are large compared with available machine memory. Instead, one may calculate and diagonalize the covariance matrix directly without ever forming the catalog matrix. For this purpose we still recommend an SVD algorithm.

Once the eigenspectra were defined, Connolly et al. projected individual galaxy spectra onto them; a classification scheme based on projection coefficients for the first two eigenspectra produced a spectral type that was strongly, but not perfectly, correlated with morphology. It should be noted that their eigenspectra are most responsive to the level of broadband continuum flux and to the discontinuities at 3600 and $4000 \AA$. In contrast, our eigenspectra are not sensitive to broadband continuum flux, as a result of high-pass filtering. The high-pass filter does admit signal from the $4000 \AA$ break, which appears as a sawtooth profile, hence the continuum break enters our analysis through its variability over a small number of frequency channels (and not through the absolute broadband flux on either side of the break). This suggests that a benefit of high-pass filtering is sensitivity to key continuum features with less stringent demands on the accuracy of absolute flux measurements.

In contrast Zaritsky et al. (1995) focused on line features. They defined a three-dimensional space based on ratios of line emission and absorption, projection onto stellar templates of young (spectral type A) and old (K) stars, and the ratio of hydrogen to oxygen emission lines. A "training set" of spectra enabled the authors to determine the loci of points in this space that correspond to morphological types. They tested the distance from these loci as measures of type using an independent set of galaxy spectra, and they concur with Connolly et al. that the correlation between spectral and morphological types is strong but not perfect. Like Connolly et al., Zaritsky et al. make the important point that the lack of perfect correlation is not the result of flaws in the spectral classification scheme but rather reflects an intrinsic property of galaxies.

We emphasize that the present classification scheme is high-pass filtered and is more sensitive to line features than to the shape of the continuum. In this regard our approach is qualitatively similar to the method of Zaritsky et al. Indeed, two of their classification parameters, the ratio of absorption to emission features and the relative line strengths of hydrogen and oxygen, correspond directly to the first two eigenspectra presented in Figure 1. The third parameter used by Zaritsky et al., a combination of projection coefficients with A-star and $\mathrm{K}$-star templates, evidently 
has relatively small galaxy-to-galaxy variance (see their Fig. 3 ); this parameter is not highly effective as a type discriminator.

Recently, Kurtz \& Mink (1998) devised a classification scheme that is based on direct estimates of the effects of survey selection, instrumental response, and the astrophysics of line formation to model template spectra without recourse to a statistical approach such as the method used here. They found that their data could be described simply and accurately by a linear combination of an absorption spectrum and an emission spectrum, with the following physical interpretation: All galaxies exhibit the absorption spectrum generated by their constituent stellar populations, but many also have superposed emission features from physically distinct $\mathrm{H}$ II regions. Thus spectral classes may be derived simply from projection onto the emission-line template. A large fraction of the $\sim 2000$ galaxies in their survey has spectra that do not project strongly onto this template; the peak in the distribution of projection coefficients for our first eigenspectrum reflects just this behavior, although in Figure $2 \mathrm{a}$ fair amount of scatter is evident about the peak.

Kurtz \& Mink have thus developed the idea that spectral classification can be efficiently accomplished with a single parameter associated with star formation. An early incarnation of this idea came from Aaronson (1978), who considered the relative contribution of red and blue stars in galaxies. To a fair approximation, the mix of $\mathrm{A}$ and $\mathrm{K}$ stars can account for variations in the broadband colors of galaxies (see Bershady 1995 for a refinement of this idea).

Despite the existence of essentially one-parameter classification methods based on the amount of star formation, virtually all schemes, including the one introduced here, have a working dimensionality of two or more, reflecting the difficulty in precisely quantifying the gamut of emission from star-forming regions with a single template. An alternative might be to project galaxy spectra onto a "variable template" (i.e., a large set of fixed templates) that reflects subtle changes in spectral profiles as a function of star formation rate. Here we essentially form such a variable template by working with a linear combination of the first two eigenspectra.

Given the similarities between our classification scheme and those for which morphological types have been considered, it is likely that the clans and morphological type are strongly correlated. Thus we adopt the nomenclature of "early" and "late" types to refer to galaxies solely on the basis of clan membership, with low clan number corresponding to early types. We now proceed to consider the clan dependence of galaxy properties; the focus here is primarily on the luminosity function.

\section{LUMINOSITY FUNCTIONS}

In their comprehensive review, Binggeli, Sandage, \& Tammann (1988) point out that the luminosity function of galaxies, $\Phi$, given the distribution of galaxies as a function of absolute magnitude $M$, should be defined in terms of galaxy types. They argue, as have others (Holmberg 1958 seems to be the first), that galaxies of different morphological type have different luminosity functions. Thus, we write the general luminosity function of the full LCRS catalog as

$$
\Phi_{g}(M)=\sum_{c=1}^{6} f_{c} \Phi_{c}(M)
$$

where $\Phi_{c}$ is the luminosity function for the $c^{\text {th }}$ clan, and $f_{c}$ is the fraction of all galaxies of the $c^{\text {th }}$ clan in some specified region of the universe. Here we treat all luminosity functions as probability distributions, hence $\Phi_{g}$ and $\Phi_{c}$ are normalized to unity upon integration over all absolute magnitude values in the range of interest:

$$
\int_{M_{\text {bri }}}^{M_{\mathrm{dim}}} d M \Phi(M)=1
$$

In our analysis $M_{\mathrm{dim}}=-16.5$ and $M_{\mathrm{bri}}=-23.0$.

In principle, there can exist a universal luminosity function that applies to all galaxies without any dependence on local environment. A case can been made against universality, and we offer some ammunition for it here. Binggeli et al. provide the starting point, the assumption that while the general luminosity function is not universal, the luminosity functions of the individual clans are. Then, one can argue against universality if the clan luminosity functions differ from one another and if the relative clan population depends on local density as is observed (Dressler 1980). An implausible conspiracy would be required to maintain universality when the luminosity function varies with galaxy type and the fraction of each type varies with local density. As a first application of the spectral classification scheme presented here, we address these issues of type dependence. We begin with an outline of the methodology.

\subsection{Method of Estimation}

We estimate luminosity functions for the LCRS galaxies using a procedure that is almost identical to that described in LC2. First, we apply the nonparametric estimator proposed by Efstathiou, Ellis, \& Peterson (1988) to obtain the unconstrained form of the luminosity functions. Then we fit the data with a Schechter function,

$$
\Phi(M)=C 10^{-0.4(\alpha+1)\left(M_{*}-M\right)} \exp \left[-10^{0.4\left(M_{*}-M\right)}\right],
$$

where $\alpha$ and $M_{*}$ correspond to the slope of the faint end of the galaxy distribution and to a typical absolute magnitude, respectively, and $C$ is a normalization constant,

$$
C=\left|\frac{1}{\Gamma\left[\alpha+1,10^{-0.4\left(M_{*}-M\right)}\right]}\right|_{M=M_{\mathrm{bri}}}^{M_{\mathrm{dim}}} .
$$

Note that absolute magnitudes are calculated on the basis of relativistic luminosity distances with cosmological deceleration parameter $q_{0}=0.5$. As in $\mathrm{LC} 2$, the results presented here are insensitive to the choice of $q_{0}$ in the range $0.1-0.5$. To obtain physical quantities such as luminosity and space density, we must also fix the Hubble parameter, $H_{0}=100 h \mathrm{~km} \mathrm{~s}^{-1} \mathrm{Mpc}^{-1}$. Where the quantity $h$ does not appear explicitly, it has been set to unity.

In addition to fitting the luminosity function with a parameterized form, we assess its "natural" shape with a nonparametric method. The nonparametric representation of choice is a stepwise function (first-order spline or histogram) of constant bin width $\delta M$ in magnitude:

$$
\Phi(M) \approx \sum_{n=-\infty}^{\infty} \phi_{n} W_{\mathrm{TH}}(M-n \delta M),
$$

where $\phi_{n}$ are constants and $W_{\mathrm{TH}}$ is a top-hat function of unit height and width, centered at the origin. Of course we cut 
the summation so that $n \delta M$ lies between $M_{\text {bri }}$ and $M_{\text {dim }}$, but even so, "nonparametric" evidently implies a large number of parameters.

The weights $\phi_{i}$ in equation (10) - or the parameters of any model of a luminosity function - can be determined from a maximum likelihood analysis (Sandage, Tammann, $\&$ Yahil 1978). Define $p_{i}$ to be the probability of observing the $i^{\text {th }}$ galaxy in a catalog with an absolute magnitude $M_{i}$, given that it is located at some specified redshift. This is equivalent to the fraction of such galaxies that could be observed given the catalog's minimum and maximum apparent magnitude cuts and other selection criteria. The likelihood that the full catalog of galaxies was observed, given their redshifts, absolute magnitudes, and the survey window, is just the product

$$
\mathscr{L}=\prod_{i=1}^{N} p_{i} .
$$

The form of the luminosity function can be estimated by first guessing it and then functionally deforming it to maximize the likelihood function in equation (11). If the number of parameters needed to determine the luminosity function's form is small, then a direct search of the parameter space quickly yields the best fit. Otherwise, as in the case of the nonparametric luminosity function in equation (10), the best fit can be obtained from iterative corrections using constraints from the derivative of $\mathscr{L}$ with respect to the $\phi_{i}$. Uncertainties in the best-fit luminosity function are calculated either by directly mapping relative confidence levels from the likelihood function or by calculating elements of the covariance matrix. Again, see Efstathiou et al. or LC2 for details.

One virtue of the maximum likelihood method is that it is insensitive to the density field; the probability $p_{i}$ represents the fraction of galaxies observable at a given magnitude, and this quantity is independent of density as long as the luminosity function itself is not density dependent. We are assuming for the moment that this is true, at least for the clan luminosity functions.

To complete the estimation of the general luminosity in equation (6), we need the number density of galaxies in each clan. Since density factors out of the maximum likelihood estimators, it must be estimated independently. We use the same prescription as in LC2 to get the mean number density:

$$
n=\frac{1}{V} \sum_{i=1}^{N} \frac{W_{i}}{\int_{M_{+}}^{M_{-}} d M \Phi(M)},
$$

where $V$ is the proper volume of the survey, the integral gives the luminosity selection function, and $W_{i}$ is a weight factor to account for surface brightness selection effects and variable sampling rates in the LCRS fields (see LC2, § 3.1). The limits of integration in equation (12), $M_{ \pm}$, give absolute magnitude cuts that simultaneously satisfy both the apparent and absolute magnitude limits of the survey.

\subsection{Luminosity Functions by Clan}

We apply the above procedure to the six clans and to the total population of the LCRS catalog observed with the 112 fiber spectrometers (NS112); the results are summarized in Figures 5 and 6 and in Table 1. There is a clear, systematic steepening of the faint-end slope as the galaxy type progresses from early to late. The Schechter parameterization is
TABLE 1

Luminosity Function Parameters by Clan

\begin{tabular}{crrrrl}
\hline \hline Clan & \multicolumn{1}{c}{$N$} & \multicolumn{1}{c}{$\alpha$} & $M_{*}-5 \log h$ & $\begin{array}{c}\bar{n} \times 10^{-3} \\
\left(h^{3} \mathrm{Mpc}^{-3}\right)\end{array}$ & $f_{\text {high }}{ }^{\mathrm{a}}$ \\
\hline $1 \ldots \ldots$ & 655 & $0.54 \pm 0.14$ & $-20.28 \pm 0.07$ & $0.34 \pm 0.03$ & $50.1 \%$ \\
$2 \ldots \ldots$ & 7614 & $-0.12 \pm 0.05$ & $-20.23 \pm 0.03$ & $7.1 \pm 0.6$ & 39.0 \\
$3 \ldots \ldots$ & 4667 & $-0.32 \pm 0.07$ & $-19.90 \pm 0.04$ & $9.9 \pm 1.3$ & 34.0 \\
$4 \ldots \ldots$. & 3210 & $-0.64 \pm 0.08$ & $-19.85 \pm 0.05$ & $11.5 \pm 2.1$ & 29.4 \\
$5 \ldots \ldots$. & 1443 & $-1.33 \pm 0.09$ & $-20.03 \pm 0.09$ & $8.4 \pm 2.2$ & 29.4 \\
$6 \ldots \ldots$ & 689 & $-1.84 \pm 0.11$ & $-20.01 \pm 0.14$ & $13.1 \pm 7.8$ & 29.5 \\
\hline
\end{tabular}

${ }^{a}$ Fraction of objects in each clan that are associated with high-density regions (see text).

reasonable over most of the magnitude range considered here, although the best-fit bright-end falloff may be too steep for late-type galaxies. Given that the eigenspectra used in our classification predominantly measure line emission, the trend in the faint-end slope was anticipated in LC2 from analysis of two subsets of galaxies partitioned according to the degree of $\mathrm{O}$ II line emission.

The formal errors given in Table 1 are derived without making complete correction for galaxies that were excluded from the LCRS on the basis of central surface brightness. As discussed in LC1, approximately $9 \%$ of the galaxies that meet all other selection criteria were cut from the S112 subsample as a result of low central surface brightness. The remaining galaxies were weighted to compensate for the excluded objects of similar absolute magnitude. The weights were determined by comparison with the N112 subsample, which has less stringent surface brightness selection. In the absence of direct measurements, the results presented here and in LC2 are not extrapolated to account for the $4 \%$ exclusion rate of the N112 subsample. To assess the systematic errors incurred by following this procedure, we examine the change in the faint-end slope of the clan luminosity functions from the N112 subsample with a number of surface brightness cuts and make the extrapolation to a cut rate of zero. The results suggest that the surface brightness cuts only marginally affect the luminosity functions of the first three clans. For example, the best-fit faint-end slopes of these functions change by less than $\Delta \alpha=0.03$. The cuts do affect the luminosity function of the fainter galaxies in clans 4, 5, and 6 to a greater degree; the faint-end slopes are expected to shift systematically downward by $0.07,0.12$, and 0.05 , respectively.

The effectiveness of the Schechter parameterization tempts us to fit not only the clan luminosity functions but the universal luminosity function for the whole catalog as well. Figure 7 compares the best-fit universal luminosity function from LC2 with a weighted sum of fitted clan luminosity functions, as in equation (6). Note that the faint-end slope of the LC2 fit is relatively flat, with $\alpha \sim-0.7$. In contrast, the faint-end slope of the weighted sum is formally $\alpha \sim-1.8$, which corresponds to the very late-type (clan 6) galaxies. If one were to pull the faint-end slope from the behavior of the weighted sum in the range -18.5 to -17.5 mag, then the slope would be approximately -1.1 , a value that is more in line with other redshift surveys (e.g., Marzke et al. 1994b), as discussed below in $\S 4.4$. We conclude that the Schechter parameterization of the general luminosity function is robust over a limited magnitude range near $M_{*}$ but can give highly misleading results when extrapolated to faint magnitudes. 


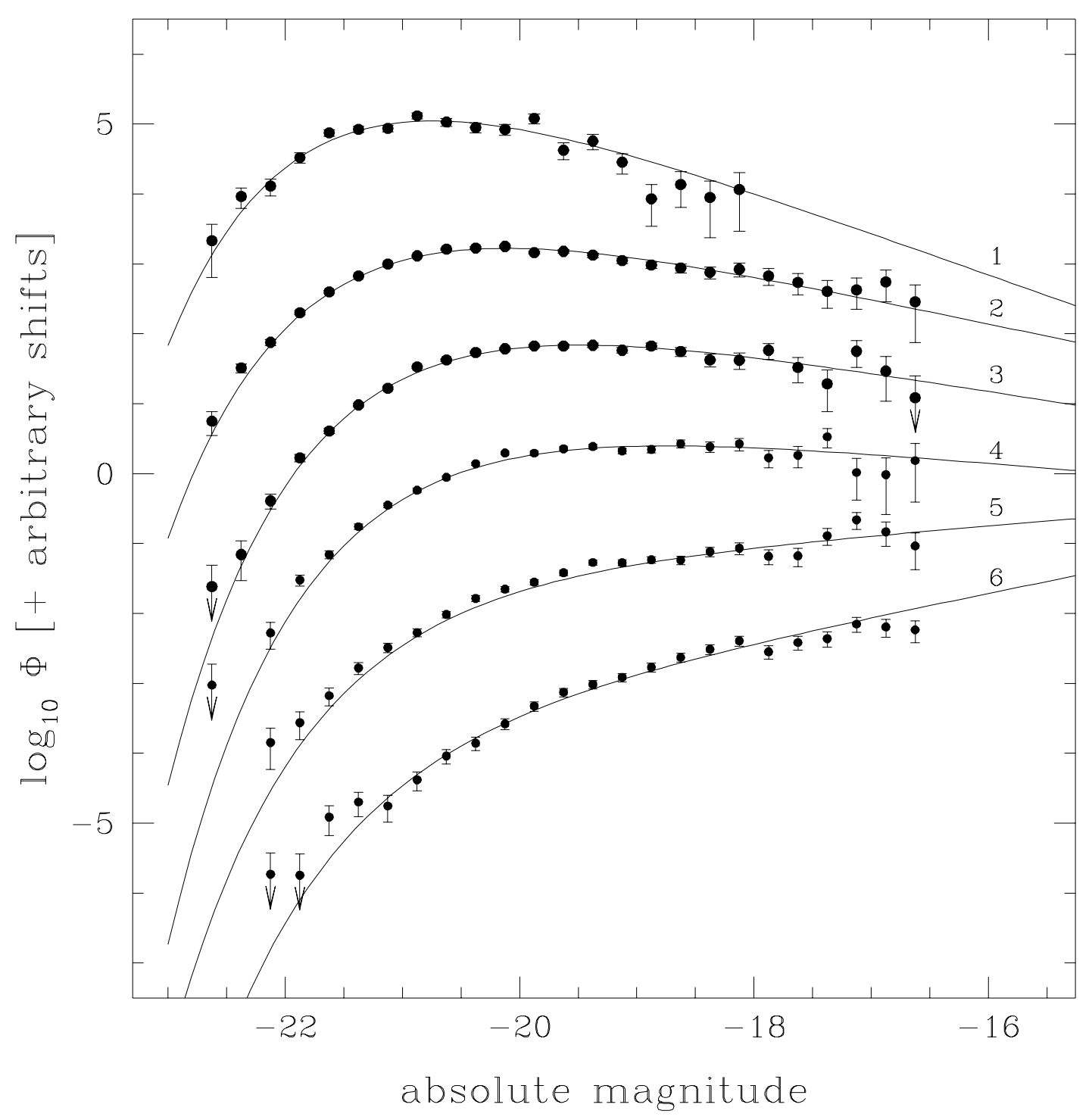

Fig. 5.-Luminosity function by clan. The points give the luminosity function from the nonparametric fit, and the curves are the best-fit Schechter functions. The points and curves have been offset in the vertical direction for clarity; the labels indicate the clan identity. Error bars show $1 \sigma$ confidence limits except in cases (arrows) where the lower limits are formally negative.

A refinement of the analysis that considers northern and southern populations separately preserves the general trend of faint-end slope with galaxy type. However, some of the clans, in particular clans 1 and 3, show significant differences in the northern and southern subsamples. A similar situation is discussed in LC2 for the estimation of a universal luminosity function from the full NS112 data: the error ellipsoids in the $\alpha-M_{*}$ plane overlap only at the $2 \sigma$ level. It is argued in LC2 that the problem arises not from the faint galaxies but from the bright ones, which can modify $\alpha$ if the Schechter parameterization gives only an approximate fit. This would suggest that the surface brightness selection of the 112 fiber data is not the cause of the discrepancy. Another possible source of the discrepancy is cosmic variance from large-scale structure and environmental effects. We examine this topic next, but we must bear the north-south differences in mind.

\subsection{Environmental Dependence}

The density-morphology relation (Dressler 1980; Postman \& Geller 1984) quantifies the extent to which the mix of galaxy types changes as a function of local density. To examine this effect in the LCRS, its galaxies may be divided into high- and low-density subsets using the threedimensional redshift space information. Of the many possible ways of making this division we opt for a friends-offriends algorithm (Huchra \& Geller 1982) to identify groups of objects in redshift space. The objective here is to simply but unambiguously determine high-density regions. We do not weigh the advantages and disadvantages of this choice except to say that while the method may misclassify some galaxies in small, high-density groups, it handles redshift space distortions-particularly "fingers of God"-very well. With link parameters of $75 \mathrm{~km} \mathrm{~s}^{-1}$ in the plane of the sky and $500 \mathrm{~km} \mathrm{~s}^{-1}$ along the line of sight and a minimum group size of four objects, the algorithm identifies about $35 \%$ of the total survey as grouped galaxies. Note that the 50 fiber objects are included in the group-finding step so that we can link members of superclusters that may span more than one $1.5 \times 1.5112$ fiber field in the survey.

Once tagged, the objects in high-density regions are tallied and compared with their low-density counterparts. 


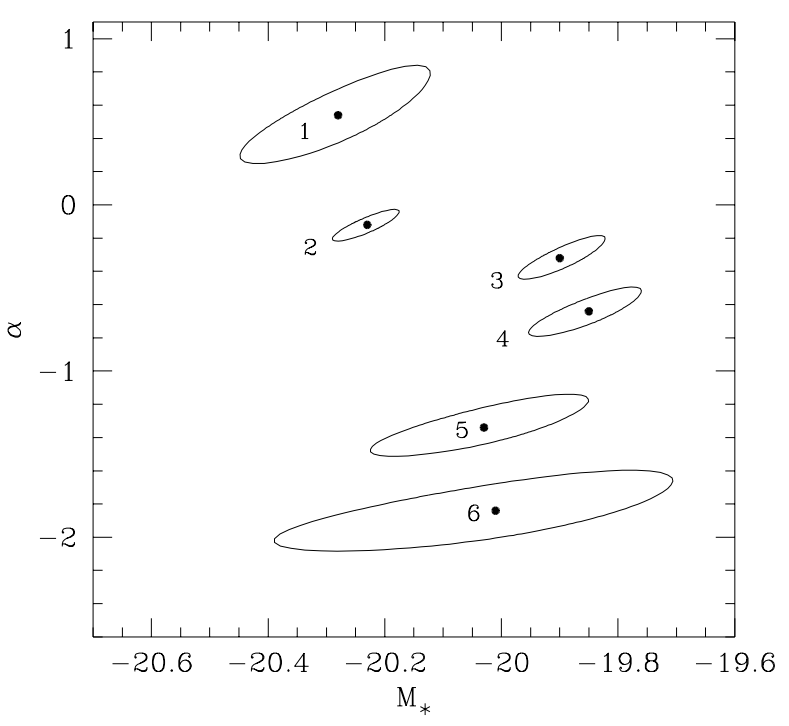

FIG. 6.-Error ellipsoids for the best-fit Schechter parameters. The labels indicate clan index, the best-fit values of $M_{*}$ and $\alpha$ are marked with solid circles, and the ellipsoids show $95 \%$ confidence intervals.
The results from raw number counts show clearly the density-morphology relation; the last column of Table 1 contains the fraction of 112 fiber members within each clan that inhabit high-density environments. Over half of the extreme early-type galaxies (clan 1) are found in regions of high density, whereas these regions contain less than a quarter of the extreme late-type (clan 6) objects. The changing mix of galaxy types with local density, although only crudely established here, clearly suggests that the general luminosity function is not universal.

The analysis may be taken a step further to determine if the luminosity functions for the individual types are universal. Using the same partition as above, we find that there is a significant density dependence in the faint-end slope, at least for the early-type objects. The effect is quite pronounced in the clan 2 galaxies, with $\alpha=0.19 \pm 0.07$ in lowdensity regions and $\alpha=-0.40 \pm 0.07$ in high-density regions. This effect and the density-morphology relation are considered in greater depth in a separate paper.

Incidentally, we have recalculated the general luminosity function $\Phi_{g}(M)$ taking into account the variation of the

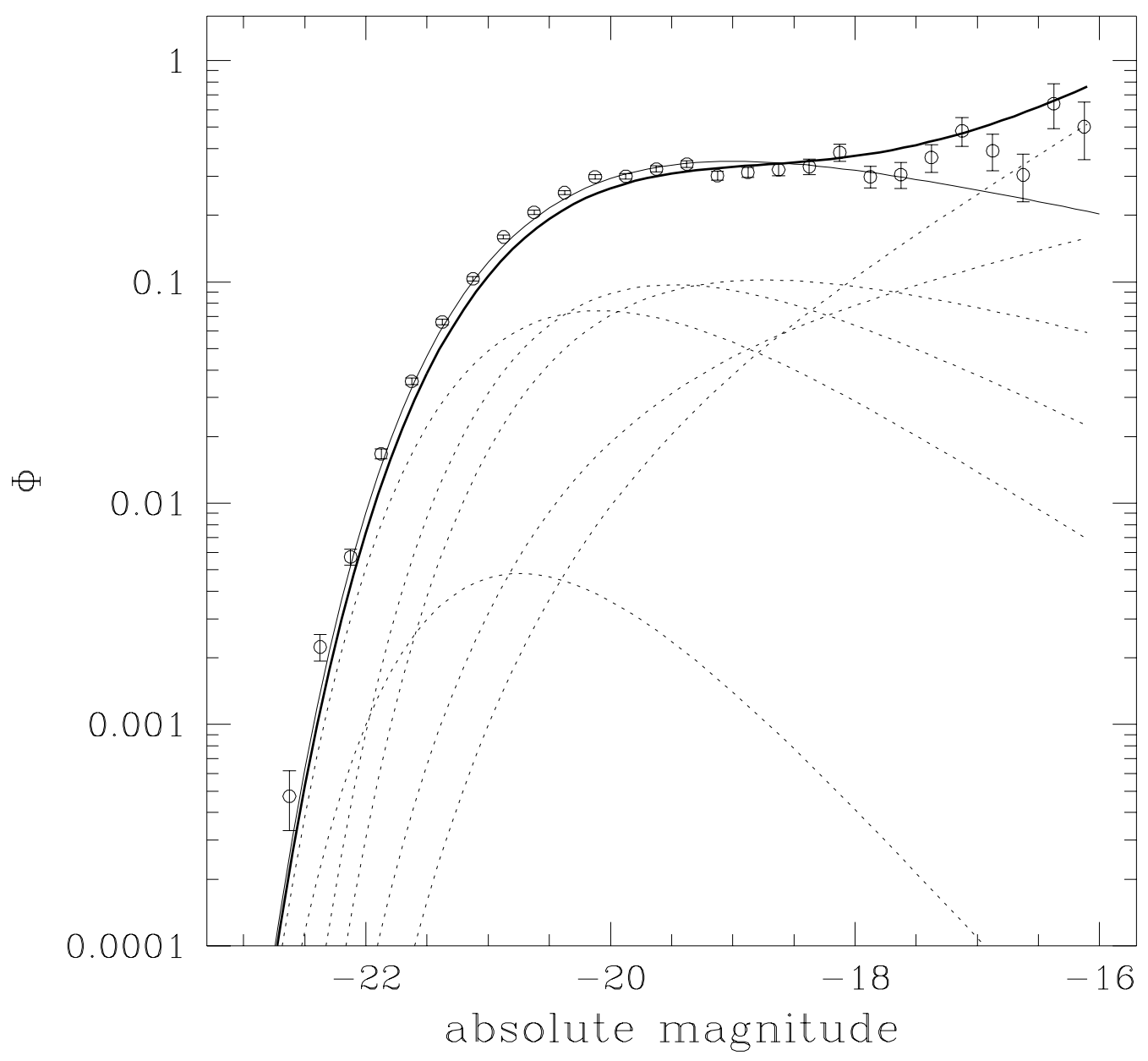

FIG. 7.- General luminosity function. The points give the luminosity function from the nonparametric fit for the full NS112 catalog, and the light solid curve is a Schechter function fit to the data. The heavy solid curve is the general luminosity function in eq. (6); the individual clan luminosity functions that contribute to it are shown as well (light dotted lines), weighted according to their contribution. The clan identity of the dotted curves can be easily determined from the faint-end slope (cf. Fig. 5). 
type-specific luminosity functions in high- and low-density regions. The result supports the conjecture by Binggeli et al. (1988) that the assumption of universality for the individual type-specific luminosity functions is still a reasonable approximation for estimating $\Phi_{g}(M)$.

\subsection{Comparison with Other Catalogs}

In many analyses of the luminosity function, the faint-end slope $\alpha$ from the Schechter parameterization has provided the means to compare different galaxy populations. In several recent examples, the value of $\alpha$ has been reported to be about -1 . For example, Loveday et al. (1992) find $\alpha=-0.97 \pm 0.15$ from the optically selected StromloAutomatic Plate Measuring Facility Redshift Survey, while Marzke, Geller, \& Huchra (1994a) report $\alpha=-1.0 \pm 2.0$ in the CfA Redshift Survey. The Autofib Redshift Survey of 1700 blue-selected galaxies (Ellis et al. 1996) and the redselected Century Survey (Geller et al. 1997) both give similar values, with $\alpha \approx-1.1$ and $\alpha \approx-1.2$, respectively.

As in the case of the LCRS, there is evidence that these reported values do not reflect the true behavior of the faint end of the general luminosity function. Marzke et al. (1994b) identify a population of Sm and Im galaxies in the CfA Survey that is expected to dominate the faint end with a slope of $\alpha \sim-1.9 \pm 0.2$. More recently, Sprayberry et al. (1997), in their examination of blue-selected low surface brightness galaxies, find a best-fit Schechter parameter of $\alpha \approx-1.4$. They also employ a model consisting of a Schechter function for magnitudes brighter than a fixed cutoff value and a power law at fainter magnitudes; the power law is constrained to match the Schechter function at the cutoff point. The power-law index $\beta$ was determined to be $\beta=-2.20$, although taken alone, the three data points that constitute "the faint end" evidently do not track such a steep power law (see their Fig. 6). Nonetheless, the data do suggest a significant excess of faint, low surface brightness galaxies over the predictions from a fit to the Schechter parameterization. Similarly Zucca et al. (1997) demonstrate that the faint end of the ESO Slice Project redshift survey is better represented by a pure power law of index $\beta \approx-1.6$, which differs significantly from the value of $\alpha \approx-1.2$ that is inferred from fitting the luminosity function near $M_{*}$. They find that this slope steepens to $\beta \approx-1.7$ for galaxies with emission lines.

Additional evidence for a steep faint-end slope comes from Driver et al. (1995), who measure galaxy number counts as a function of apparent magnitude in a deep image from the Hubble Space Telescope Wide Field Planetary Camera. They model the number count distribution of three groupings of galaxy types using the Schechter parameterization and argue that late-type objects require some combination of a steep $(\alpha \lesssim-1.5)$ faint-end slope and evolution of the luminosity function in an attempt to reconcile both the (high) number counts and the (low) local density of faint objects (e.g., Phillips \& Driver 1995).

There is no strong signal of a faint-end upturn in the general luminosity function of the Century Survey as determined by Geller et al. (1997), just as in the type-independent analysis of the LCRS in LC2. This is reassuring given the similarities between the waveband selection of the two surveys. Yet despite these similarities, there are significant differences in the best-fit Schechter parameters. The problem is likely triggered in part by the use of the Schechter parameterization in the first place: The results of $\S 4.2$ suggest that the parameterization is not well suited to describe the general luminosity function except over a narrow range of magnitudes. For example, relatively small changes in the absolute magnitude range used in a fit can significantly alter the inferred parameters. A simple change in the bright magnitude limit from $M_{\text {bri }}=-23$ to -21 decreases the best-fit $\alpha$ for the LCRS from -0.7 to -0.85 . This example suggests that the discrepancy between the LCRS and the Century Survey seems to lie at the bright end of the luminosity function and is not the result of missing faint galaxies.

Another explanation of the discrepancy between the LCRS and Century Survey results might come from the fact that the Century Survey encompasses the Corona Borealis supercluster and therefore may have a different mix of galaxy types as a result of the density-morphology relation. A better comparison with the LCRS results will be possible with the forthcoming type-dependent analysis of the Century Survey.

\section{CONCLUSION}

In this paper we have implemented a spectral classification scheme based on SVD. The SVD approach isolates the most significant variations in the spectra of a catalog with a set of orthonormal basis vectors or eigenspectra. Projection of individual galaxy spectra onto the two most significant eigenspectra yields coefficients from which galaxy types are determined. The method was applied to the LCRS, and in that catalog we defined six spectral classes (here called clans). The physical properties of each clan were interpreted in terms of star formation; the known correlation between star-forming galaxies and morphological type (Kennicutt 1992a) suggests that the clan index runs smoothly from early to late types.

The eigenspectra derived from SVD are also useful for finding galaxies with unusual spectral properties. The projection coefficients of the third eigenspectrum alone allowed us to isolate a distinct population of objects $(0.6 \%$ of the total) that we identify as Seyfert 1 galaxies.

As an application of the classification scheme presented here, we used spectral types to study the luminosity function of galaxies. A strong type dependence is identified, as manifested by the faint-end slope, with $\alpha$ ranging from about +0.5 (early types) to -1.8 (late types). We include this type dependence in the calculation of the general luminosity function, and we identify a faint-end slope that rises steeply, with a power-law index of $\sim-1.8$ in the faint limit. Furthermore, the general luminosity function exhibits a broad shoulder at an absolute magnitude of roughly -20 in the $R$ band. These two features are not both represented by a Schechter function. On the other hand, the individual typespecific luminosity functions are mostly well characterized by the Schechter parameterization. Small deviations from the model occur for the luminosity function of the extreme late-type galaxies, which show a slight excess at bright magnitudes.

The LCRS galaxies are also seen to reflect the densitymorphology relation (cf. Table 1, last column). Given the wide range of luminosity function shapes among the six galaxy types considered here, any type-independent assessment of the luminosity function may be sensitive to the large-scale structure encompassed by the survey. In addition we report preliminary evidence for variation in the type-specific luminosity function of some clans as a function 
of local density. The effect is most prominent in the (earlytype) clan 2 population.

Finally we compared the LCRS luminosity function with those of other redshift surveys. The case for a steep faint-end rise to the luminosity function, with a power-law index around -2 , is now quite strong. The lower values of faintend slope reported in the literature result primarily from poor sampling of faint galaxies, coupled with an inappropriate extrapolation of the best-fit $\alpha$-parameter. In some recent work (e.g., Zucca et al. 1997; Sprayberry et al. 1997) the excess number of faint galaxies has prompted the use of a model consisting of a Schechter function with a break at some faint magnitude to a pure power law. A similar effect can be achieved by taking the luminosity function to be a linear combination of Schechter functions with varying $\alpha$ parameters, and on the basis of the results presented here, we argue that this latter approach is more realistic.
The success of the spectral classification method used here on the LCRS galaxies suggests that it may be fruitfully applied to larger catalogs such as the upcoming Sloan Digital Sky Survey. Then, with better sky coverage we may be able to examine in greater detail the luminosity function, a critical ingredient for understanding cosmic structure formation.

We thank M. Kurtz for helpful discussions regarding spectral classification and the referee, M. Bershady for many insightful comments and suggestions that greatly enhanced the presentation and focus of this paper. W. H. P. and B. C. B. acknowledge funding from NSF grant PHY 95-07695. B. C. B. is grateful to NASA Offices of Space Sciences, Aeronautics and Mission to Planet Earth for providing computing resources.
Aaronson, M. 1978, ApJ, 221, L103

Allen, D. A., Norris, R. P., Meadows, V. S., \& Roche, P. F. 1991, MNRAS, 248,528

Baldwin, J. A., Phillips, M. M., \& Terlevich, R. 1981, PASP, 93, 5

Bershady, M. A. 1995, AJ, 109, 87

Binggeli, B., Sandage, A., \& Tammann, G. A. 1988, ARA\&A, 509

Connolly, A. J., Szalay, A. S., Bershady, M. A., Kinney, A. L., \& Calzetti, D. 1995, AJ, 110, 1071

Dressler, A. 1980, ApJ, 236, 351

Driver, S. P., Windhorst, R. A., Ostrander, E. J., Keel, W. C., Griffiths, R. E., \& Ratnatunga, K. U. 1995, ApJ, 449, L23

Efstathiou, G., Ellis, R. S., \& Peterson, B. A. 1988, MNRAS, 232, 431

Ellis, R. S., Coless, M., Broadhurst, T., Heyl, J., \& Glazebrook, K. 1996, MNRAS, 280, 235

Folkes, S. R., Lahav, O., \& Maddox, S. J. 1996, MNRAS, 283, 651

Geller, M. J., et al. 1997, AJ, 114, 220

Holmberg, E. 1958, Medd. Lund Astron. Obs., Ser. 2, 128

Huchra, J. P., \& Geller, M. J. 1982, ApJ, 257, 423

Kendall, M. G. 1975, Multivariate Analysis (London: Griffin)

Kennicutt, R. C. Jr. 1992a, ApJ, 388, 310 .1992b, ApJS, 79, 255

Kurtz, M. J., \& Mink, D. J. 1998, PASP, in press

\section{REFERENCES}

Lin, H., Kirshner, R. P., Shectman, S. A., Landy, S. D., Oemler, A., Tucker, D. L., \& Schechter, P. L. 1996, ApJ, 464, 60 (LC2)

Loveday, J., Peterson, B. A., Efstathiou, G., \& Maddox, S. J. 1992, ApJ, 390, 338

Marzke, R. O., Geller, M. J., \& Huchra, J. P. 1994a, ApJ, 428, 43

Marzke, R. O., Geller, M. J., Huchra, J. P., \& Corwin, H. G. 1994b, AJ, 108, 437

Morgan, W. W., \& Mayall, N. V. 1957, PASP, 69, 291

Odewahn, S. C., Windhorst, R. A., Driver, S. P., \& Keel, W. C. 1996, ApJ, 472, L13

Phillipps, S., \& Driver, S. P. 1995, MNRAS, 274, 832

Postman, M., \& Geller, M. J. 1984, ApJ, 281, 95

Press, W. H., Teukolsky, S. A., Vetterling, W. T., \& Flannery, B. P. 1992, Numerical Recipes (Cambridge: Cambridge Univ. Press)

Roberts, M. S., \& Haynes, M. P. 1994, ARA\&A, 32, 115

Sandage, A., Tammann, G. A., \& Yahil, A. 1979, ApJ, 232, 352

Shectman, S. A., Landy, S. D., Oemler, A., Tucker, D. L., Lin, H., Kirshner, R. P., \& Schechter, P. L. 1996, ApJ, 470, 172 (LC1)

Sprayberry, D., Impey, C. D., Irwin, M. J., \& Bothun, G. D. 1997, ApJ, 482, 104

Zaritsky, D., Zabludoff, A. I., \& Willick, J. A. 1995, AJ, 110, 1602

Zucca, E., et al. 1997, A\&A, 326, 477 